\title{
Двухконтактные тандемные солнечные элементы DSC/c-Si: оптимизация параметров фотоэлектрода на основе диоксида титана
}

\author{
(C) А.Б. Никольская, М.Ф. Вильданова, С.С. Козлов, О.И. Шевалеевский
}

Институт биохимической физики им. Н.М. Эмануэля Российской академии наук, 119334 Москва, Россия

E-mail: anickolskaya@mail.ru

(Получена 11 апреля 2017 г. Принята к печати 17 апреля 2017 г.)

Сконструированы и исследованы новые типы двухконтактных тандемных солнечных элементов на основе $\mathrm{DSC} / c-\mathrm{Si}$, в которых тонкопленочный солнечный элемент на основе сенсибилизированного красителем мезоскопического слоя диоксида титана (DSC) был соединен с солнечным элементом на основе кристаллического кремния $(c-\mathrm{Si})$ по параллельной схеме. Измерены оптические и фотоэлектрические параметры отдельных элементов и тандемных элементов DSC/c-Si на их основе. Показано, что максимальное значение кпд фотопреобразования для тандемного солнечного элемента DSC $/ c-\mathrm{Si}$ в условиях стандартного освещения AM1.5G $\left(100 \mathrm{MBT} / \mathrm{cm}^{2}\right)$ было достигнуто при использовании в DSC фотоэлектрода на основе диоксида титана толщиной 3.5 мкм и составило $14.7 \%$.

DOI: $10.21883 /$ FTP.2018.01.45325.8591

\section{1. Введение}

Солнечные элементы (СЭ) на основе сенсибилизированных красителем мезоскопических слоев диоксида титана или DSC (dye-sensitized solar cells) являются предметом многочисленных исследований и технологических разработок последнего десятилетия [1,2]. Основными преимуществами фотопреобразователей типа DSC являются простая технология изготовления, низкая стоимость и, что существенно, способность этого типа СЭ сохранять высокие кпд в условиях низкой и диффузной освещенности [3-5]. Повышенные результаты эффективности фотопреобразования в условиях стандартного освещения AM1.5G (100 мBт/см²) показали DSC, сконструированные на основе допированных слоев диоксида титана [6,7]. Рекордное значение эффективности в элементах данного типа составило 13\% [8], что, однако, значительно уступает значениям кпд, достигнутым в СЭ на основе кремния (c-Si) или халькопирита (CIGS) [9].

Одним из направлений работ по повышению эффективности DSC является создание различных типов тандемных систем. Реализация простой схемы тандемного солнечного элемента (ТСЭ) на основе сочетания двух типов DSC с взаимодополняющими спектрами поглощения привела к несущественному повышению кпд элемента [10]. Более успешной оказалась идея создания TCЭ, где DSC использовался в качестве верхнего СЭ в сочетании с нижним СЭ на основе CIGS [11].

Следует отметить, что в научной литературе по солнечной фотовольтаике слабо освещен вопрос эффективности различных типов СЭ в условиях низкой солнечной инсоляции и при диффузном освещении. При этом в реальных условиях эксплуатации СЭ и солнечных панелей, особенно в широтах средней и северной России, а также Европы, это имеет первостепенное значение при выборе оптимальных типов фотопреобразователей [12]. В связи с этим одной из наиболее оптимальных тандемных схем, с нашей точки зрения, является тандемный СЭ, в котором верхний элемент адаптирован к эффективной работе в условиях низкой инсоляции. Перспективной конструкцией подобного ТСЭ является тандемный фотопреобразователь DSC/c-Si [13].

В последнее десятилетие в литературе по фотовольтаике тандемные элементы на основе DSC и органических CЭ принято подразделять на двухконтактные и четырехконтактные ТСЭ [14]. Первые представляют собой класс ТСЭ, в котором верхний и нижний элементы соединены параллельно или последовательно между собой и имеют на выходе два электрических контакта, во втором случае каждый из элементов снабжен независимыми электрическими выходами $[15,16]$.

В нашей работе представлены результаты по конструированию и исследованию работы двухконтактного TCЭ, в котором DSC и CЭ типа $c$-Si были соединены между собой по параллельной схеме. Использование при конструировании TCЭ DSC с фотоэлектродами различной толщины и различной степенью оптического пропускания позволило оптимизировать конструкцию ТСЭ типа DSC $/ c-\mathrm{Si}$ и получить максимальные значения кпд преобразования солнечной энергии в электрическую.

\section{2. Методика эксперимента}

\section{1. Солнечные элементы типа DSC}

В качестве подложек для конструирования DSC были использованы специальные стекла Solaronix размером $2 \times 2$ см с высоким оптическим пропусканием $(T>80 \%)$, покрытые тонким проводящим слоем на основе оксида олова, допированного фтором (FTO) с проводимостью $\sim 10$ Ом/см². Подложки предварительно были очищены в ультразвуковой ванне в растворе детергента, затем в растворах этанола и ацетона. Далее на них по известной ранее методике $[6,17]$ с использованием 
метода „медицинской бритвы“ тонким слоем наносилась паста $\mathrm{TiO}_{2}$ с последующим отжигом в печи при $500^{\circ} \mathrm{C}$ в течение 30 мин. Для приготовления пасты использовался коммерческий порошок $\mathrm{TiO}_{2}$ Degussa Aeroxide® P25 c преимущественным содержанием анатаза, который смешивался с терпинеолом, этилцеллюлозой и этанолом.

В результате были приготовлены три серии СЭ типа DSC на основе мезоскопических слоев $\mathrm{TiO}_{2}$ различной толщины $d(d=3.5,6.1$ и 10.1 мкм $)$ и рабочей площадью каждого $\sim 0.7 \times 0.7 \mathrm{~cm}^{2}$. Сенсибилизация фотоэлектродов проводилась в течение 24 ч в смеси ацетонитрила и трет-бутилового спирта в соотношении $1: 1$, содержащей краситель N719 (рутениевый комплекс сложной структуры) в концентрации 0.3 мМ [7]. В качестве платинового противоэлектрода использовалась стеклянная подложка, на которую методом распыления наносился раствор $\mathrm{H}_{2} \mathrm{PtCl}_{6}$ (2 мг $\mathrm{Pt}$ в 1 мл этанола) с последующим отжигом при $400^{\circ} \mathrm{C}$ в течение 15 мин [7]. При окончательной сборке CЭ типа DSC электрод и противоэлектрод накладывались друг на друга с последующей герметизацией по периметру с помощью пленки Surylin 1702. Далее пространство между электродами через заранее просверленные в противоэлектроде отверстия заполнялось электролитом (раствор AN-50 Iodolyte, Solaronix)

\section{2. Конструирование TCЭ типа DSC/c-Si}

На рис. 1 представлена блок-схема ТСЭ типа $\mathrm{DSC} / c$-Si, где в качестве верхнего элемента использовался DSC с варьируемой толщиной мезоскопического слоя $\mathrm{TiO}_{2}$, а в качестве нижнего - CЭ на основе $c$-Si размером $1.7 \times 2.1$ см. Образцы $c$-Si были любезно предоставлены исследовательской группой Г.Г. Унтилы из НИИЯФ МГУ им. М.В. Ломоносова.

Солнечные элементы в составе двухконтактной тандемной системы были электрически соединены между собой по параллельной схеме, а их плоскости были ориентированы нормально к направлению светового потока. ТСЭ был смонтирован на общей стеклянной подложке, которая использовалась в качестве несущей конструкции. Чтобы в экспериментальных измерениях освещаемая область нижнего $c$-Si CЭ соответствовала рабочей площади DSC $\left(0.7 \times 0.7 \mathrm{~cm}^{2}\right)$, поверх кремниевого образца накладывалась соответствующая маска.

\section{3. Методы исследования}

Исследование морфологии мезоскопического слоя $\mathrm{TiO}_{2}$ и его толщины проводилось методом сканирующей электронной микроскопии (SEM) на установке FEI Helios NanoLab 660i (СШA).

Абсорбционные спектры для мезоскопических слоев $\mathrm{TiO}_{2}$, сенсибилизированных красителем N719, были получены на двулучевом UV-vis спектрофотометpe Shimadzu 3600 с интегрирующей сферой ISR-3100 (Япония).

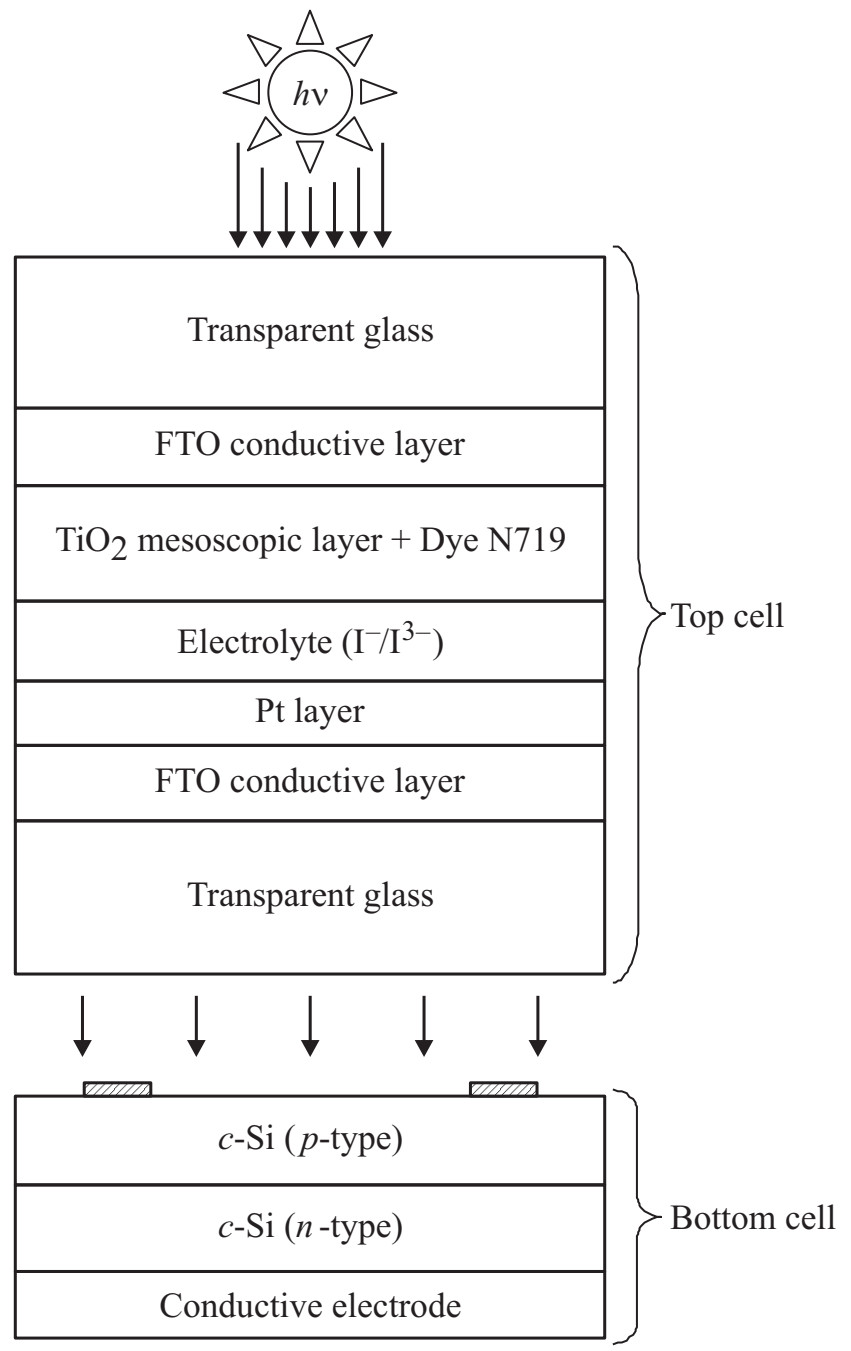

Рис. 1. Блок-схема двухконтактного ТCЭ $\mathrm{DSC} / c$-Si.

Исследование работы CЭ типа DSC и c-Si, а также TCЭ на их основе проводилось в условиях стандартного освещения AM1.5G с использованием солнечного имитатора Abet 10500 (Abet Technologies, CША). Вольт-амперные характеристики (BAX) записывались на универсальном анализаторе 4200-SCS (Keithley, CША). Спектры квантовой эффективности СЭ, IPCE (incident photon-to-current conversion efficiency), исследовались с использованием автоматизированной системы QEX10 (PV Measurments, CША) в диапазоне длин волн $300-1200$ HM.

\section{3. Экспериментальные результаты}

Согласно данным SEM значение толщины нанесенных на проводящие подложки мезоскопических слоев $\mathrm{TiO}_{2}(d)$ для трех серий приготовленных образцов составило 3.5, 6.1 и 10.1 мкм соответственно. На рис. 2 в качестве примера приведена микрофотография по- 
перечного среза фотоэлектрода $\mathrm{TiO}_{2}$ с $d=6.1$ мкм. Полученные мезоскопические слои характеризовались однородностью и отсутствием дефектов.

На рис. 3 приведены оптические спектры пропускания для мезоскопических слоев $\mathrm{TiO}_{2}$, сенсибилизированных красителем N719. Степень поглощения света в них была пропорциональна толщине электрода. Максимальная абсорбция наблюдалась при толщине слоя $d=10.1$ мкм.

На основании полученных данных по пропусканию и отражению в приготовленных слоях $\mathrm{TiO}_{2}$, следуя описанной ранее методике [18], были рассчитаны коэффициенты поглощения, из спектральных зависимостей которых были вычислены значения ширины запрещенной зоны $\left(E_{g}\right)$ для слоя $\mathrm{TiO}_{2}$. Полученное значение составило величину 3.06 эВ, которая не зависела от толщины слоя $\mathrm{TiO}_{2}$, что хорошо согласуется с литературными данными [18].

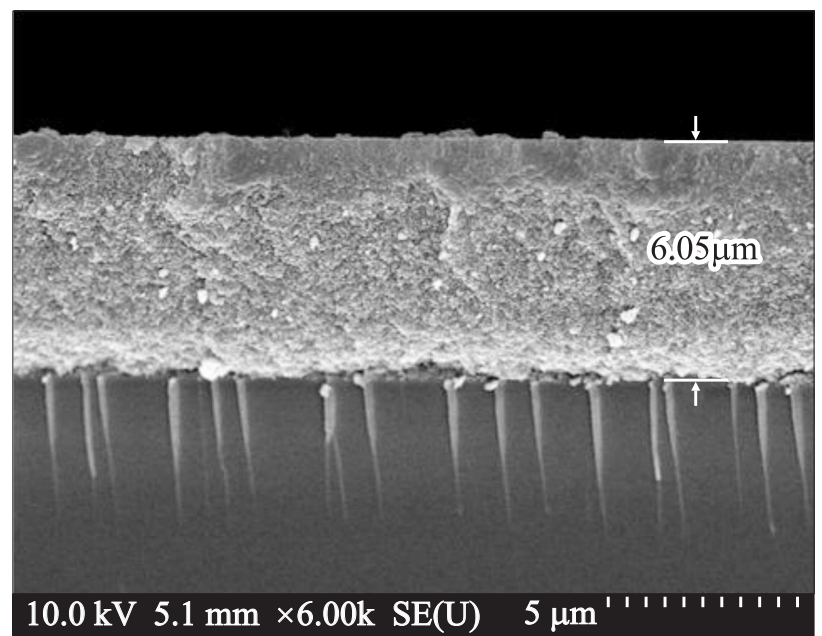

Рис. 2. Электронная микрофотография поперечного среза мезоскопического слоя $\mathrm{TiO}_{2}$ толщиной 6.1 мкм, нанесенного на подложку с проводящим FTO покрытием.

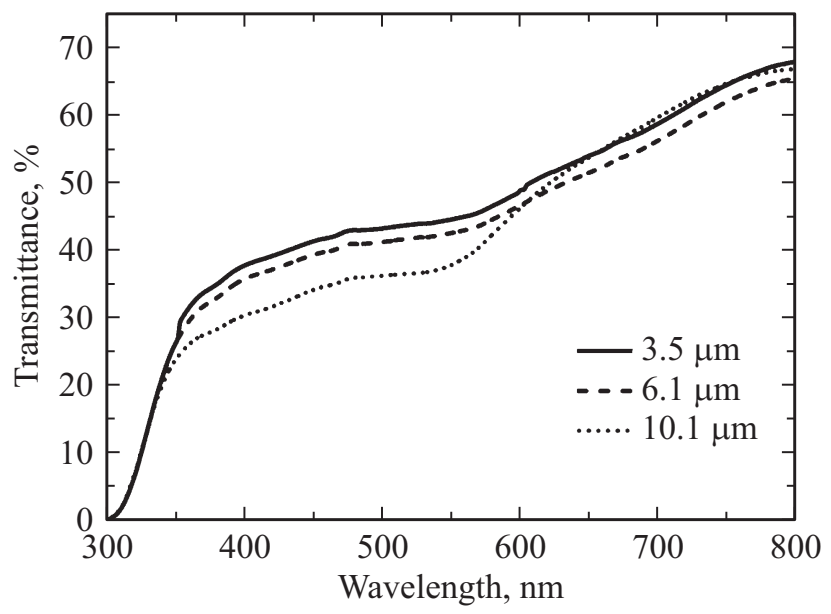

Pис. 3. Спектры оптического пропускания сенсибилизированных красителем N719 мезоскопических слоев $\mathrm{TiO}_{2}$ различной толщины.

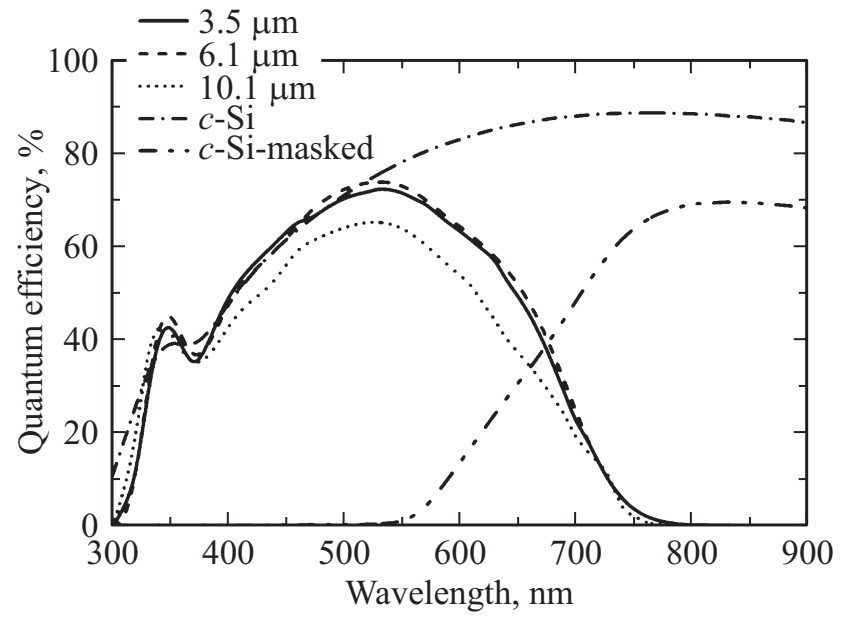

Рис. 4. Спектральные характеристики внешней квантовой эффективности СЭ на основе DSC с различной толщиной мезоскопического слоя $\mathrm{TiO}_{2}$, а также для $\mathrm{C} Э c$-Si и $c$-Si-masked.

После сборки образцов DSC для каждого из сконструированных СЭ были получены спектры IPCE, записаны зависимости BAX и вычислены значения эффективности преобразования.

На рис. 4 представлены спектральные зависимости внешней квантовой эффективности для образцов DSC с различной толщиной мезоскопического слоя $\mathrm{TiO}_{2}$. Квантовая эффективность в СЭ типа DSC существенным образом не зависела от толщины мезоскопического слоя $\mathrm{TiO}_{2}$; некоторое снижение наблюдалось только для слоя $\mathrm{TiO}_{2}$ с $d=10.1$ мкм. Для сравнения на рис. 4 приведены также спектральные характеристики IPCE для СЭ на основе $c$-Si и для $c$-Si-masked, где элемент $c$-Si освещался через DSC с толщиной $\mathrm{TiO}_{2} d=3.5$ мкм. Видно, что наиболее эффективное фотопреобразование в DSC происходит в интервале длин волн от 400 до 600 нм, тогда как в кремниевом СЭ максимальная квантовая эффективность наблюдается в диапазоне 500-900 нм. Таким образом, сочетание СЭ на основе мезоскопических слоев $\mathrm{TiO}_{2}$ и $c$-Si позволяет максимально утилизировать спектральную область видимого света.

Фотоэлектрические параметры для CЭ DSC и TCЭ DSC/c-Si с различной толщиной мезоскопического слоя $\mathrm{TiO}_{2}:(1)-3.5$, (2) - 6.1, (3) - 10.1 мкм, а также для СЭ $c$-Si и $c$-Si-masked

\begin{tabular}{l|c|c|c|r}
\hline & $J_{\mathrm{SC}}, \mathrm{MA} / \mathrm{cm}^{2}$ & $V_{\mathrm{OC}}, \mathrm{B}$ & $F F$ & $\eta, \%$ \\
\cline { 2 - 5 } DSC (1) & 17.69 & 0.785 & 0.53 & 7.4 \\
DSC (2) & 20.23 & 0.785 & 0.54 & 8.6 \\
DSC (3) & 15.22 & 0.790 & 0.55 & 6.6 \\
$c$-Si & 37.83 & 0.545 & 0.61 & 12.6 \\
$c$-Si-masked & 23.90 & 0.530 & 0.60 & 7.6 \\
DSC $(1) / c$-Si & 40.88 & 0.580 & 0.62 & 14.7 \\
DSC $(2) / c-S i$ & 38.67 & 0.575 & 0.62 & 13.8 \\
DSC $(3) / c-S i$ & 28.51 & 0.580 & 0.61 & 10.1
\end{tabular}




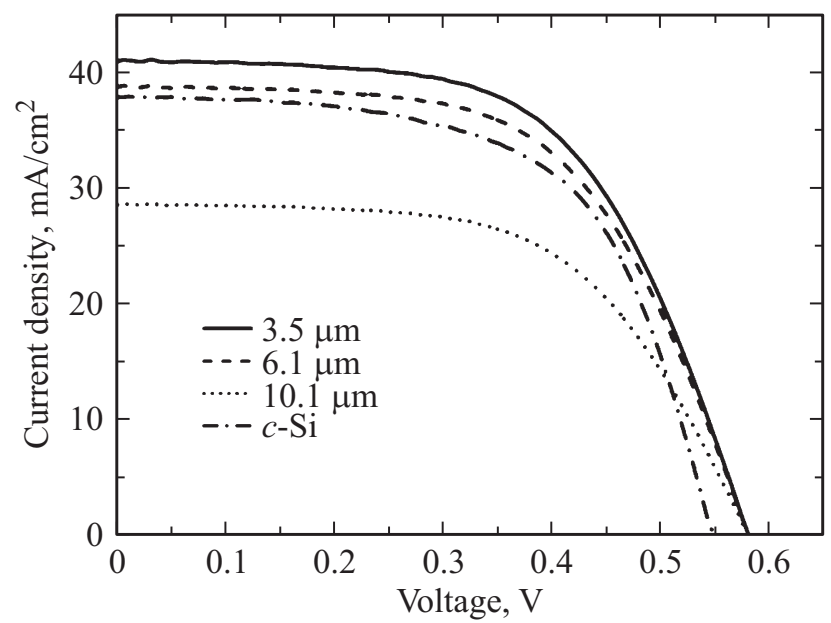

Рис. 5. ВАХ для ТСЭ $\mathrm{DSC} / c-\mathrm{Si}$ с различной толщиной мезоскопического слоя $\mathrm{TiO}_{2}$ и для $\mathrm{C} Э c$-Si.

BAX и значения кпд, полученные для СЭ типа DSC и $c-\mathrm{Si}$, а также для ТCЭ типа DSC/c-Si, представлены на рис. 5 и в таблице соответственно. Кпд фотопреобразования $\eta(\%)$ рассчитывался по известной формуле [19]:

$$
\eta=\frac{J_{\mathrm{SC}} V_{\mathrm{OC}} F F}{P_{\mathrm{IN}}} \cdot 100 \%,
$$

где $J_{\mathrm{SC}}-$ плотность тока короткого замыкания, $V_{\mathrm{OC}}-$ напряжение холостого хода, $F F$ - коэффициент заполнения, $P_{\mathrm{IN}}-$ интенсивность освещения.

Как видно из таблицы, для DSC с толщиной $\mathrm{TiO}_{2}$ $d=6.1$ мкм фотоэлектрические параметры достигают максимальных значений, в то время как в DSC с $d=10.1$ мкм они минимальны. Последнее может свидетельствовать о небольшой, сравнимой с величиной $d$, длине диффузии носителей заряда через слой полупроводника [20]. Поведение спектров IPCE для всех образцов DSC было практически идентичным (рис. 4), из чего можно сделать вывод о том, что процесс диффузии носителей заряда из объема мезоскопического слоя $\mathrm{TiO}_{2}$ к проводящей подложке является основным фактором, лимитирующим работу СЭ рассматриваемого типа. Следует заметить, что для толщины мезоскопического слоя полупроводника в DSC $d=3.5$ мкм величины токов оказались ниже, чем при $d=6.1$ мкм. Это обусловлено тем, что в первом случае в объеме слоя $\mathrm{TiO}_{2}$ в процессе сенсибилизации абсорбируется меньшее число молекул красителя, ответственных за поглощение квантов света. Таким образом, в DSC элементе $(d=6.1$ мкм) реализуется оптимальное соотношение толщины слоя фотоэлектрода, что позволяет получить максимальные значения эффективности в тандемном двухконтактном элементе DSC/c-Si.

Все три серии образцов DSC с различной толщиной мезоскопического слоя $\mathrm{TiO}_{2}$ были использованы в качестве верхних элементов при конструировании ТСЭ в сочетании $c$-Si. BAX, приведенные на рис. 5, показывают, что во всех случаях, кроме DSC с $d=10.1$ мкм, применение тандемной схемы позволяет значительно повысить токи короткого замыкания. Величины напряжения холостого хода при этом практически не меняются.

Приведенные на рис. 5 и в таблице данные показывают, что при освещении образцов светом с интенсивностью $100 \mathrm{MBT} / \mathrm{cm}^{2}$ наиболее высокие значения кпд фотопреобразования (14.7\%) в ТСЭ были достигнуты при сочетании кремниевого CЭ с DSC, у которого толщина мезоскопического слоя $\mathrm{TiO}_{2} d=3.5$ мкм. При этом ТСЭ, сконструированный с использованием наиболее эффективного DSC $(d=6.1$ мкм), показал меньшее значение кпд (13.8\%). Последнее обусловлено абсорбционными характеристиками верхнего элемента: чем меньше толщина поглощающего слоя в CЭ типа DSC, тем больше света доходит до нижнего элемента на основе $c$-Si (cм. рис. 3).

Суммируя вышеизложенное, можно сказать, что эффективность фотопреобразования света в электричество в TCЭ типа DSC/c-Si существенно зависит не только от кпд верхнего элемента, но и от его оптических характеристик. В рассмотренном случае определяющим параметром является толщина фотоэлектрода на основе $\mathrm{TiO}_{2}$ в DSC, оптимизация которой позволяет получить максимальные значения эффективности тандемного фотопреобразователя.

\section{4. Заключение}

Были сконструированы и исследованы новые типы двухконтактных ТСЭ на основе сочетания солнечных элементов типа DSC и $c$-Si. Представлены спектры квантовой эффективности отдельных СЭ и вольт-амперные характеристики изготовленных ТСЭ, полученные в условиях стандартного освещения АM1.5G. Оптимизация толщины мезоскопического слоя $\mathrm{TiO}_{2}$ в DSC, которая в данном случае составила 3.5 мкм, позволила достичь максимального значения кпд в тандемном фотопреобразователе $\mathrm{DSC} / c-\mathrm{Si}$, равного $14.7 \%$. Полученные результаты указывают на перспективность конструирования ТСЭ с использованием DSC в качестве верхнего элемента: технологически простое изменение толщины мезоскопического слоя позволяет регулировать интенсивность потока солнечного излучения, доходящего до нижнего СЭ тандемной структуры.

Работа выполнена при частичной поддержке РФФИ, гранты № 16-08-00620, 16-29-06416.

\section{Список литературы}

[1] M. Grätzel. J. Photochem. Photobiol. C Photochem. Rev., 4 (2), 145 (2003).

[2] O. Shevaleevskiy. Pure Appl. Chem., 80 (10), 2079 (2008).

[3] B.E. Hardin, H.J. Snaith, M.D. McGehee. Nature photonics 6, 162 (2012). 
[4] K.G. Reddy, T.G. Deepak, G.S. Anjusree, S. Thomas, S. Vadukumpully, K.R.V. Subramanian, S.V. Nair, A.S. Nair. Phys. Chem. Chem. Phys., 16, 6838 (2014).

[5] M.F. Vildanova, A.B. Nikolskaia, S.S. Kozlov, O.I. Shevaleevskiy. J. Phys. Conf., 643, 012106 (2015).

[6] N. Tsvetkov, L. Larina, O. Shevaleevskiy, B.T. Ahn. Prog. Photovolt.: Res. Appl., 20, 904 (2012).

[7] S. Kozlov, A. Nikolskaia, L. Larina, M. Vildanova, A. Vishnev, O. Shevaleevskiy. Phys. Status Solidi A, 213 (7), 1801 (2016).

[8] S. Mathew, A. Yella, P. Gao, R. Humphry-Baker, B.F.E. Curchod, N. Ashari-Astani, I. Tavernelli, U. Rothlisberger, M.K. Nazeeruddin, M. Grätzel. Nat. Chem., 6, 242 (2014).

[9] M.A. Green, K. Emery, Y. Hishikawa, W. Warta, E.D. Dunlop, D.H. Levi, A.W.Y. Ho-Baillie. Prog. Photovolt.: Res. Appl., 25, 3 (2017).

[10] M. Durr, A. Bamedi, A. Yasuda, G. Nelles. Appl. Phys. Lett., 84 (17), 3397 (2004).

[11] P. Liska, K.R. Thampi, M. Graetzel, D. Bremaud, D. Rudmann, H.M. Upadhyaya, A.N. Tiwari. Appl. Phys. Lett., 88, 203103 (2006).

[12] C. Cornaro, S. Bartocci, D. Musella, C. Strati, A. Lanuti, S. Mastroianni, S. Penna, A. Guidobaldi, F. Giordano, E. Petrolati, T.M. Brown, A. Reale, A. Di Carlo. Prog. Photovolt.: Res. Appl., 23, 215 (2015).

[13] Заявка на патент № 2013120153 от 06.05.2013.

[14] G. Dennler, N.S. Sariciftci, C.J. Brabec. Encyclopedia of Materials: Science and Technology (The Netherlands, Elsevier Ltd., 2010) p. 1.

[15] C.D. Bailie, M.G. Christoforo, J.P. Mailoa, A.R. Bowring, E.L. Unger, W.H. Nguyen, J. Burschka, N. Pellet, J.Z. Lee, M. Grätzel, R. Noufi, T. Buonassisi, A. Salleo, M.D. McGehee. Energy Environ. Sci., 8, 956 (2015).

[16] T. Duong, N. Lal, D. Grant, D. Jacobs, P. Zheng, S. Rahman, H. Shen, M. Stocks, A. Blakers, K. Weber, T.P. White, K.R. Catchpol. IEEE J. Photovolt., 6, 679 (2016).

[17] N. Tsvetkov, L. Larina, O. Shevaleevskiy, B.T. Ahn. Energ. Environ. Sci., 4, 1480 (2011).

[18] A. Hagfeldt, G. Boschloo, L. Sun, L. Kloo, H. Pettersson. Chem. Rev., 110, 6595 (2010).

[19] A. Luque, S. Hegedu. Handbook of Photovoltaic Science and Engineering (Chichester, John Wiley \& Sons Ltd., 2003) p. 92.

[20] S. Södergren, A. Hagfeldt, J. Olsson, S.-E. Lindquist. J. Phys. Chem., 98, 5552 (1994).

Редактор Г.А. Оганесян
Two-terminal tandem solar cells DSC $/ c$-Si: optimization of $\mathrm{TiO}_{2}$-based photoelectrode parameters

\author{
A.B. Nikolskaia, M.F. Vildanova, S.S. Kozlov, \\ O.I. Shevaleevskiy
}

Institute of Biochemical Physics,

Russian Academy of Sciences,

119334 Moscow, Russia

Abstract New types of two-terminal tandem solar cells $\mathrm{DSC} / c$-Si in which mesoscopic dye-sensitized solar cell (DSC) was connected in parallel with a silicon crystalline $(c-\mathrm{Si})$ solar cell, were developed and investigated. We have measured the optical and photovoltaic parameters for both the individual and the fabricated tandem $\mathrm{DSC} / c-\mathrm{Si}$ solar cells. It was shown that the highest efficiency of $14.7 \%$ for the tandem $\mathrm{DSC} / c$-Si solar cell under standard AM1.5G illumination conditions was achieved for DSC based on $3.5 \mu \mathrm{m}$ thick titanium dioxide photoelectrode. 